\title{
Construction and analysis of a ceRNA network and patterns of immune infiltration in bladder cancer
}

\author{
Yang $\mathrm{Fu}^{1}{ }^{\wedge}$, Shanshan Sun ${ }^{2}$ Jianbin $\mathrm{Bi}^{1}$, Chuize Kong ${ }^{1}$, Lei Yin ${ }^{1}$ \\ ${ }^{1}$ Department of Urology, The First Hospital of China Medical University, Shenyang, China; ${ }^{2}$ Department of Pharmacy, The First Hospital of China \\ Medical University, Shenyang, China \\ Contributions: (I) Conception and design: Y Fu, L Yin; (II) Administrative support: J Bi, C Kong, L Yin; (III) Collection and assembly of the data: \\ Y Fu, S Sun; (V) Data analysis and interpretation: Y Fu, S Sun; (VII) Manuscript writing: All authors; (VII) Final approval of the manuscript: All \\ authors. \\ Correspondence to: Prof. Chuize Kong, PhD, MD; Dr. Lei Yin, PhD, MD. Department of Urology, The First Hospital of China Medical University, \\ No. 155 Nanjing North Street, Heping District, Shenyang 110001, Liaoning Province, China. Email: kongchuize_cmu@sina.cn; yinleicmu@163.com.
}

Background: Bladder cancer (BC) is the ninth most common malignant tumor, accounting for an estimate of 549,000 new BC cases and 200,000 BC-related deaths worldwide in 2018. The prognosis of BC has not substantially improved despite significant advances in the diagnosis and treatment of the disease.

Methods: The RNA sequencing (RNA-seq) data and clinical information of BC patients were downloaded from The Cancer Genome Atlas (TCGA) database. The Cell-type Identification By Estimating Relative Subsets Of RNA Transcripts (CIBERSORT) algorithm was used to assess immune infiltration. The survival analyses were performed using the selected components of a ceRNA network and selected immune cell types by least absolute shrinkage and selection operator (LASSO) Cox regression to calculate the risk score. The accuracy of prognosis prediction was determined by receiver operating characteristic (ROC) curves, survival curves, and nomograms. Finally, the correlation analysis was performed to investigate the relationships between the signature components of the ceRNA network and the immune cell signature.

Results: Two completed survival analyses included selected components of the ceRNA network (ELN, SREBF1, DSC2, TTLL7, DIP2C, SATB1, hsa-miR-20a-5p, and hsa-miR-29c-3p) and selected immune cell types (M0 macrophages, M2 macrophages, resting mast cells, and neutrophils). ROC curves, survival curves (all $\mathrm{P}$ values $<0.05$ ), nomograms, and calibration curves indicated that the accuracy of the two survival analyses was acceptable. Moreover, the correlations between TTLL7 and resting mast cells $(\mathrm{R}=0.24$, $\mathrm{P}<0.001)$, DSC2 and resting mast cells $(\mathrm{R}=-0.23, \mathrm{P}<0.001), \mathrm{ELN}$ and resting mast cells $(\mathrm{R}=0.44, \mathrm{P}<0.001)$, and hsa-miR-29c-3p and M0 macrophages $(\mathrm{R}=-0.29, \mathrm{P}<0.001)$ were significant, indicating that interactions of these factors may play significant roles in the prognosis of $\mathrm{BC}$.

Conclusions: TTLL7, DSC2, ELN, hsa-miR-29c-3p, resting mast cells, and M0 macrophages may play an important role in the development of BC. However, additional studies are needed to confirm this hypothesis.

Keywords: Bladder cancer (BC); The Cancer Genome Atlas (TCGA); ceRNA; immune cell infiltration; risk score

Submitted Sep 09, 2020. Accepted for publication Mar 09, 2021.

doi: $10.21037 /$ tau-20-1250

View this article at: http://dx.doi.org/10.21037/tau-20-1250

^ ORCID: 0000-0003-3770-5074. 


\section{Introduction}

Bladder cancer (BC) is the ninth most common malignant tumor worldwide (1), accounting for an estimate of 549,000 new BC cases and 200,000 BC-related deaths in 2018 (2). $\mathrm{BC}$ is classified into non-muscle invasive bladder cancer (NMIBC) and muscle invasive bladder cancer (MIBC), and transitional cell carcinoma is the most common pathological type $(3,4)$. NMIBC accounts for $75 \%$ of BC cases, and $50 \%$ of NMIBC cases progress to MIBC (5). Transurethral resection of bladder tumor (TURBt) is considered the main treatment for NMIBC, and radical cystectomy is the strategy for MIBC treatment (6). However, current treatment strategies for $\mathrm{BC}$ have not significantly improved the 5 -year survival rate over the past decade (7). Moreover, $\mathrm{BC}$ has become one of the most expensive to treat solid tumors due to high recurrence rate (8). Therefore, it is essential to identify biomarkers that can accurately evaluate the diagnosis, treatment, and prognosis of BC.

Noncoding RNAs (ncRNAs), including long noncoding RNAs (lncRNAs) and microRNAs (miRNAs), have been recently recognized as the key regulatory factors in tumors (9). Overexpression of lncRNA DLX6 antisense RNA 1 (DLX6-AS1) and insulin-like growth factor binding protein 4-1 (IGFBP4-1) may lead to a poor prognosis in BC patients $(10,11)$. Upregulation of miRNA-133b and miR-375-3p inhibits the proliferation and metastasis of BC cells $(12,13)$. The occurrence of tumors is closely related to interactions between lncRNAs, mRNAs, and miRNAs (14). LncRNAs and mRNAs can competitively bind miRNAs by miRNA response elements (MREs) to generate a competitive endogenous RNA (ceRNA) network to regulate the expression of various RNAs and proteins $(15,16)$. This ceRNA network can be used to investigate the functions of lncRNAs and to identify biomarkers related to the diagnosis and prognosis of $\mathrm{BC}$, which is essential for assisting the clinicians with early diagnosis, risk assessment, and appropriate treatment decision-making for patients with BC $(17,18)$.

Immunotherapy has become a promising antitumor strategy that recognizes tumors as foreign antigens and inhibits the proliferation and metastasis of the tumor cells by inducing active or passive immune responses $(19,20)$. In BC, Bacillus Calmette-Guerin vaccine (BCG) suppresses tumor cells by activating immune cell infiltration and is the gold standard for therapy of high-risk NMIBC $(21,22)$. However, approximately $40 \%$ of BC patients do not respond to $\mathrm{BCG}$, and $15 \%$ of $\mathrm{BC}$ patients experience progression to MIBC after the treatment $(23,24)$. Recent studies have shown that immune-related genes were significantly associated with the prognosis of cancer. High expression of ENDRA was associated with lower survival rate in patients with advanced $\mathrm{BC}$ (25). Overexpression of RAC3 promoted the migration of cancer cells and was associated with a decrease in the recurrence-free survival in ER3-positive breast cancer (26). The exploration of immune checkpoint inhibitors (ICIs) facilitated great progress in immunotherapy. In phase II clinical trials, the neoadjuvant use of ICIs resulted in pathological complete responses in patients with MIBC (27).

A number of recent studies demonstrated important roles of the ceRNA networks and immune cell infiltration in evaluation of prognosis in patients with malignant tumors $(28,29)$. Some studies suggested that a combination of a ceRNA network and immune cell infiltration has potential value in cancer research $(30,31)$. Therefore, we constructed a ceRNA network for BC using the data downloaded from The Cancer Genome Atlas (TCGA) database. Two survival analyses were performed, including analysis based on selected genes included in the ceRNA network and analysis based on the data on immune cell infiltration estimated by the Cell-type Identification By Estimating Relative Subsets Of RNA Transcripts (CIBERSORT) algorithm. These two survival analyses were used to identify the key factors and immune cells that can accurately predict patients with BC, and these factors were combined for subsequent analysis, which is very important for early diagnosis and treatment of BC. Finally, the relationships between these two survival analyses were analyzed (Figure 1). The article is presented in accordance with the STROBE reporting checklist (available at http://dx.doi.org/10.21037/tau-20-1250).

\section{Methods}

\section{Identification of differentially expressed genes (DEGs)}

The data for $411 \mathrm{BC}$ tissues and 19 normal tissues were downloaded from the TCGA dataset (The Cancer Genome Atlas) (https://portal.gdc.cancer.gov/) and used for mRNA and lncRNA analysis. The data for $418 \mathrm{BC}$ tissues and 19 normal tissues were downloaded from the TCGA dataset and used for miRNA analysis. The clinical data of 409 patients with $\mathrm{BC}$ were obtained from TCGA (including age, gender, grade, stage, and TNM classification) (Table 1). The DESeq2 method was used to identify DEGs, including lncRNAs, miRNAs, and mRNAs. DESeq2 can improve the stability and 


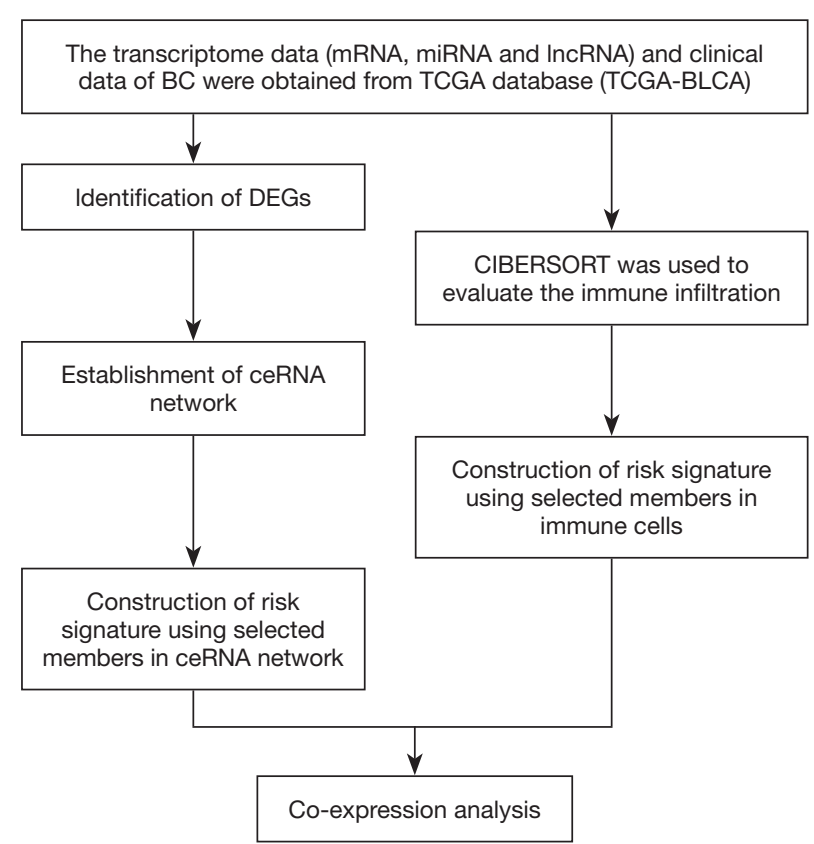

Figure 1 The flow diagram of the present study. BC, bladder cancer; TCGA, The Cancer Genome Atlas; DEGs, differentially expressed genes; ceRNA, competitive endogenous RNA; CIBERSORT, cell-type identification by estimating relative subsets of RNA transcripts.

interpretability of estimation fold change (FC) and dispersion by shrinkage estimators $(32,33)$. The data corresponding to duplicate and abnormal samples (not primary tumors and abnormal samples) were deleted, and the remaining data were normalized by the trimmed mean of M-values (TMM) method implemented in edgeR and further transformed by the voom method provided in limma. TMM normalization is a simple and effective method for estimation of relative RNA levels using RNA sequencing (RNA-seq) data. Moreover, voom is faster and more convenient than existing methods of RNA-seq analysis and converts RNA-seq data into a form that can be analyzed using tools similar to those used for microarray analysis. The cutoff criteria were defined as $\mid \log 2$ $\mathrm{FCl}>1.0$ and false discovery rate (FDR) adjusted $\mathrm{P}<0.05$. Ethical approval was not required because the data used in the present study were obtained from public databases. The study was conducted in accordance with the Declaration of Helsinki (as revised in 2013).

\section{Construction of a ceRNA network}

StarBase was introduced to predict the lncRNA-miRNA
Table 1 Characteristics of the included BC patients obtained from the TCGA database

\begin{tabular}{|c|c|}
\hline Basic information & Total $(n=409)$ \\
\hline Age & 69 (median) \\
\hline \multicolumn{2}{|l|}{ Gender } \\
\hline Female & 106 \\
\hline Male & 303 \\
\hline \multicolumn{2}{|l|}{ Grade } \\
\hline High & 385 \\
\hline Low & 21 \\
\hline Unknow & 3 \\
\hline \multicolumn{2}{|l|}{ Stage } \\
\hline$|\&| \mid$ & 132 \\
\hline III \& IV & 275 \\
\hline Unknow & 2 \\
\hline \multicolumn{2}{|l|}{ T classification } \\
\hline $\mathrm{T} 1 \& \mathrm{~T} 2$ & 124 \\
\hline T3 \& T4 & 253 \\
\hline TX & 1 \\
\hline Unknow & 31 \\
\hline \multicolumn{2}{|l|}{$\mathrm{N}$ classification } \\
\hline No & 237 \\
\hline N1 \&N2 \& N3 & 131 \\
\hline NX & 36 \\
\hline Unknow & 5 \\
\hline \multicolumn{2}{|l|}{ M classification } \\
\hline MO & 194 \\
\hline M1 & 11 \\
\hline $\mathrm{MX}$ & 202 \\
\hline Unknow & 2 \\
\hline
\end{tabular}

BC, bladder cancer; TCGA, the The Cancer Genome Atlas.

and miRNA-mRNA interactions (34). StarBase (http:// starbase.sysu.edu.cn/) is an open-source database that can display extensive and complex RNA-RNA (miRNAlncRNA, miRNA-pseudogene, miRNA-circRNA, and miRNA-mRNA) and protein-RNA interaction networks by analyzing millions binding sites of Argonaute (Ago) and other RNA-binding protein (RBP) identified by 
crosslinking immunoprecipitation and high-throughput sequencing (CLIP-seq) (35). Interactions with $\mathrm{P}$ values $<0.05$ for the hypergeometric and correlation tests were considered significant. We used Cytoscape software version 3.8.0 to display the ceRNA network.

\section{Survival analysis based on the components of the ceRNA network}

All components of the ceRNA network were incorporated into a univariate Cox regression model to identify the genes related to survival $(\mathrm{P}<0.05)$. The survival analysis of the components of the ceRNA network was performed by least absolute shrinkage and selection operator (LASSO) Cox regression to avoid overfitting (36). The median value of the risk score calculated by LASSO was used as the cutoff value to divide patients into the high-risk and the low-risk groups. Kaplan-Meier survival curves were compared using the logrank test and a nomogram to assess the survival outcomes [overall survival (OS)]. A receiver operating characteristic (ROC) curve and a calibration curve were constructed to evaluate the accuracy of the risk score.

\section{Immune cell infiltration}

We used CIBERSORT to evaluate the immune infiltration data for each BC sample downloaded from TCGA. CIBERSORT is a deconvolution algorithm that can predict the abundance of 22 immune cell types based on the gene expression data $(37,38)$.

\section{Survival analysis based on selected immune cells}

All immune cells were incorporated into a univariate Cox regression model to identify immune cells related to survival $(\mathrm{P}<0.05)$. The survival analysis based on immune cells was performed by LASSO Cox regression to avoid overfitting. The patients were divided into the high-risk and low-risk groups based on the median value of the risk score. KaplanMeier survival curves were compared using the log-rank test and a nomogram to assess the survival outcomes (OS). A ROC curve and a calibration curve were constructed to evaluate the accuracy of the risk score. Finally, Pearson correlation analysis was used to assess the correlations between various immune cells and between the components of the immune cell signature and the components of the ceRNA network signature.

\section{Multidimensional validation}

Multidimensional verification was performed using a series of external databases, including Gene Expression Omnibus (GEO) (https://www.ncbi.nlm.nih.gov/geo/), the Gene Expression Profiling Interactive Analysis (GEPIA), and TargetScan database (http://www.targetscan.org/), to reduce possible errors. We also downloaded the relevant immunohistochemical images from the public database Human Protein Atlas database (HPA) (https://www. proteinatlas.org/) to detect the gene expression in tumor tissues and normal tissues, but no specific staining method was provided in the database.

\section{Statistical analysis}

All statistical analyses were performed using $\mathrm{R} 3.53$ software. A ceRNA network was constructed using the GDCRNATools package of R (39). The log-rank test and univariate Cox regression analyses were performed using the survival package of $\mathrm{R}$. The survival time and status of the patients were assumed to be dependent variables, and the expression of the components of the ceRNA network and immune cell infiltration were assumed to be independent variables in Cox regression used for two survival analyses. LASSO Cox regression was performed using the glmnet package of R. ROC curves were generated and plotted using the survivalROC package of $\mathrm{R}$. Nomograms and calibration curves were generated using the rms package of $\mathrm{R}$. The differences in the gene expression levels were evaluated by the Mann-Whitney U test.

\section{Results}

\section{DEGs}

We identified 2,581 differentially expressed mRNAs (1,261 upregulated and 1,320 downregulated mRNAs), 223 differentially expressed miRNAs (152 upregulated and 71 downregulated miRNAs), and 216 differentially expressed lncRNAs (39 upregulated and 177 downregulated lncRNAs) in BC versus normal tissues (Figure 2A,B,C).

\section{Construction of a ceRNA network and survival analysis}

We constructed a ceRNA network composed of 110 mRNAs, 15 miRNAs, and 7 lncRNAs (Figure 3). Then, all components of the ceRNA network were incorporated into 

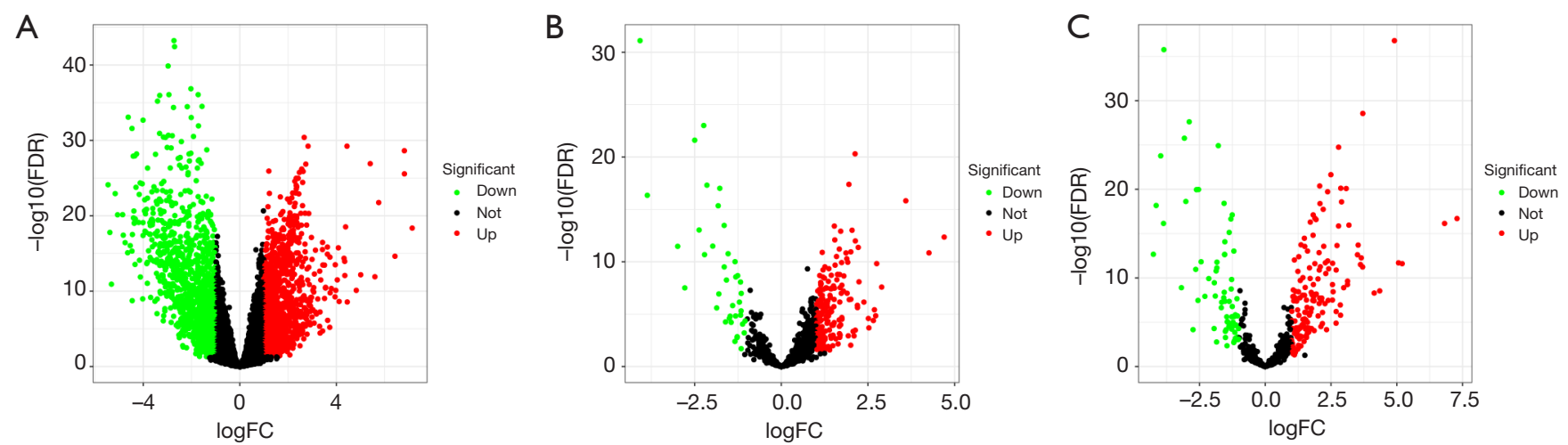

Figure 2 DEGs. Differentially expressed mRNAs (A), lncRNAs (B), and miRNAs (C) were identified in BC. Red dots represent upregulated genes, and green dots represent downregulated genes. DEGs, differentially expressed genes; BC, bladder cancer.

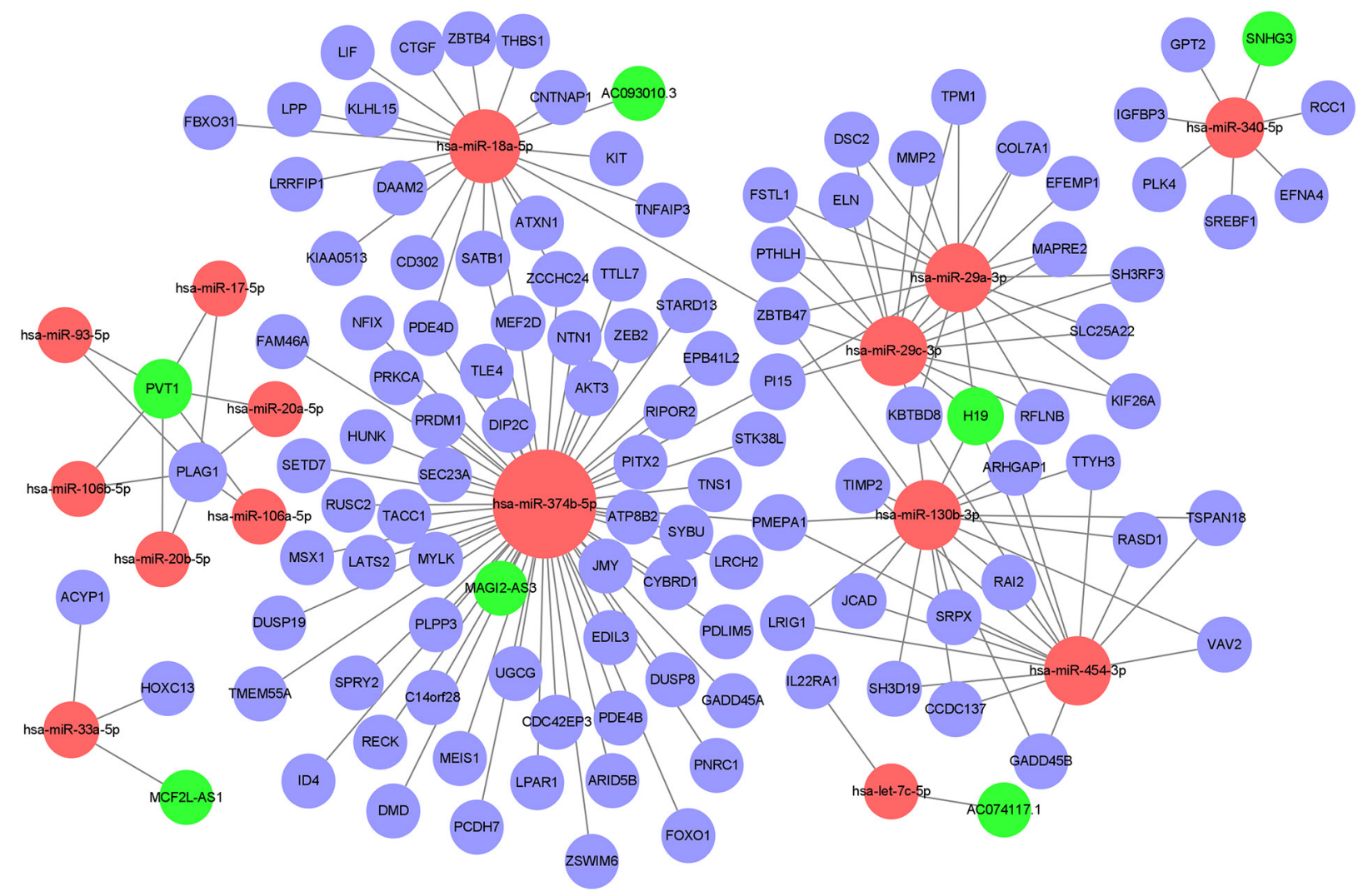

Figure 3 A ceRNA network. We constructed a ceRNA network using differentially expressed mRNAs, lncRNAs, and miRNAs. Purple ellipses represent mRNAs, green ellipses represent lncRNAs, and red ellipses represent miRNAs. The size of red ellipses corresponds to the number of the edges. CeRNA, competitive endogenous RNA. 
Table 2 The genes related to survival identified by univariable Cox regression

\begin{tabular}{|c|c|c|c|c|}
\hline Gene & $\mathrm{HR}$ & $95 \% \mathrm{Cl}$ (low) & $95 \% \mathrm{Cl}$ (high) & $P$ \\
\hline TIMP2 & 1.142 & 1.045 & 1.247 & 0.003 \\
\hline$E L N$ & 1.131 & 1.054 & 1.214 & 0.001 \\
\hline NTN1 & 1.099 & 1.024 & 1.179 & 0.009 \\
\hline MYLK & 1.109 & 1.025 & 1.200 & 0.010 \\
\hline KIF26A & 1.154 & 1.047 & 1.273 & 0.004 \\
\hline CYBRD1 & 1.120 & 1.015 & 1.236 & 0.024 \\
\hline SREBF1 & 1.174 & 1.021 & 1.349 & 0.024 \\
\hline TNS1 & 1.102 & 1.010 & 1.202 & 0.030 \\
\hline PTHLH & 1.064 & 1.011 & 1.119 & 0.017 \\
\hline SEC23A & 1.424 & 1.165 & 1.740 & 0.001 \\
\hline SRPX & 1.129 & 1.047 & 1.218 & 0.002 \\
\hline RASD1 & 1.101 & 1.011 & 1.200 & 0.026 \\
\hline EFEMP1 & 1.119 & 1.056 & 1.186 & 0.000 \\
\hline AKT3 & 1.128 & 1.015 & 1.253 & 0.026 \\
\hline CTGF & 1.121 & 1.032 & 1.217 & 0.007 \\
\hline RECK & 1.147 & 1.021 & 1.288 & 0.021 \\
\hline PMEPA1 & 1.143 & 1.050 & 1.244 & 0.002 \\
\hline ATXN1 & 1.163 & 1.015 & 1.334 & 0.030 \\
\hline$D S C 2$ & 1.113 & 1.041 & 1.191 & 0.002 \\
\hline Pl15 & 1.100 & 1.023 & 1.183 & 0.010 \\
\hline THBS1 & 1.145 & 1.038 & 1.264 & 0.007 \\
\hline$T T L L 7$ & 1.175 & 1.069 & 1.291 & 0.001 \\
\hline TPM1 & 1.185 & 1.060 & 1.325 & 0.003 \\
\hline ATP8B2 & 1.235 & 1.105 & 1.381 & 0.000 \\
\hline LRIG1 & 1.160 & 1.065 & 1.264 & 0.001 \\
\hline SETD7 & 1.252 & 1.019 & 1.538 & 0.032 \\
\hline$D A A M 2$ & 1.139 & 1.022 & 1.269 & 0.019 \\
\hline LATS2 & 1.232 & 1.042 & 1.457 & 0.015 \\
\hline DIP2C & 1.322 & 1.103 & 1.584 & 0.003 \\
\hline$M S X 1$ & 1.162 & 1.050 & 1.285 & 0.004 \\
\hline FSTL1 & 1.192 & 1.053 & 1.350 & 0.005 \\
\hline EDIL3 & 1.121 & 1.013 & 1.241 & 0.027 \\
\hline $\mathrm{ZCCHC24}$ & 1.135 & 1.018 & 1.265 & 0.023 \\
\hline$J C A D$ & 1.123 & 1.008 & 1.251 & 0.036 \\
\hline
\end{tabular}

Table 2 (continued)
Table 2 (continued)

\begin{tabular}{|c|c|c|c|c|}
\hline Gene & HR & $95 \% \mathrm{Cl}$ (low) & $95 \% \mathrm{Cl}$ (high) & $\mathrm{P}$ \\
\hline MAPRE2 & 1.181 & 1.025 & 1.360 & 0.021 \\
\hline ZEB2 & 1.124 & 1.003 & 1.259 & 0.044 \\
\hline $\mathrm{PCDH7}$ & 1.068 & 1.001 & 1.139 & 0.045 \\
\hline SH3RF3 & 1.164 & 1.041 & 1.302 & 0.008 \\
\hline ZBTB4 & 1.349 & 1.037 & 1.754 & 0.026 \\
\hline SATB1 & 0.853 & 0.761 & 0.956 & 0.006 \\
\hline RFLNB & 1.205 & 1.050 & 1.382 & 0.008 \\
\hline DUSP8 & 1.122 & 1.003 & 1.255 & 0.044 \\
\hline RUSC2 & 1.160 & 1.005 & 1.339 & 0.042 \\
\hline AC074117.1 & 0.746 & 0.607 & 0.918 & 0.006 \\
\hline hsa-let-7c-5p & 1.161 & 1.067 & 1.264 & 0.001 \\
\hline hsa-miR-106b-5p & 0.795 & 0.642 & 0.985 & 0.036 \\
\hline hsa-miR-17-5p & 0.835 & 0.706 & 0.988 & 0.036 \\
\hline hsa-miR-20a-5p & 0.804 & 0.688 & 0.939 & 0.006 \\
\hline hsa-miR-29c-3p & 0.846 & 0.745 & 0.961 & 0.010 \\
\hline hsa-miR-93-5p & 0.775 & 0.653 & 0.919 & 0.003 \\
\hline
\end{tabular}

a univariate Cox regression model to identify components related to survival $(\mathrm{P}<0.05)$ (Table 2). The survival analysis by LASSO Cox regression used the selected components (Figure 4A,B; Table 3). The median value of the risk score (1.072) calculated by LASSO was used as the cutoff value to divide $\mathrm{BC}$ patients into the high-risk and low-risk groups. ROC curve showed that OS of BC patients was perfectly predicted by the risk score [area under the curve (AUC) for 1 -year survival $=0.690$; AUC for 3 -year survival $=0.707$; and AUC for 5 -year survival $=0.743$ ] (Figure $4 C$ ). The prognosis of $\mathrm{BC}$ patients in the high-risk group was worse than that in the low-risk group $(\mathrm{P}<0.001)$ (Figure $4 D)$. A nomogram predicted the probability of 1-, 3-, and 5-year OS (Figure $4 E$ ). The calibration curve indicated that prediction using the nomogram was accurate (Figure $4 F$ ).

\section{Immune cell infiltration}

CIBERSORT was used to evaluate the immune infiltration data for each BC sample downloaded from TCGA, and bar plots and heatmaps were used to visualize the data 
A

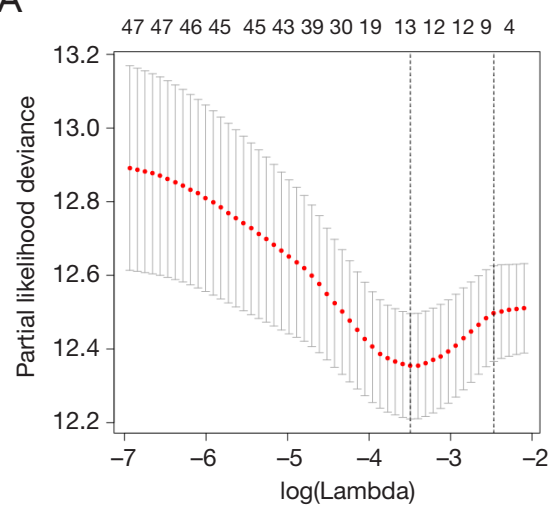

D
B

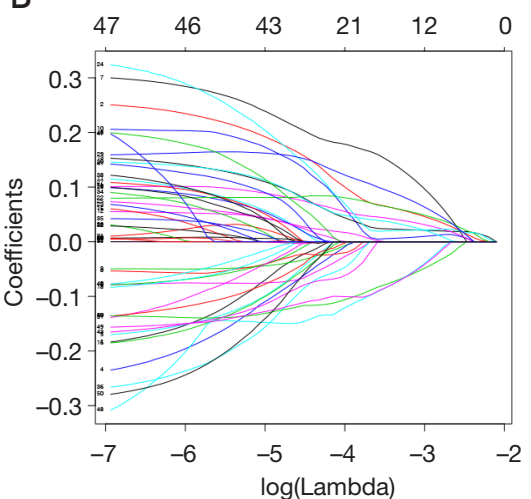

E
C

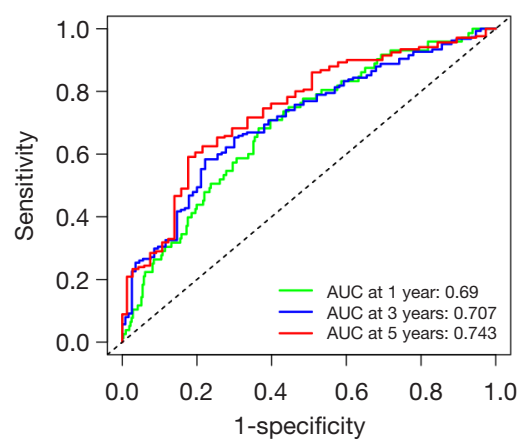

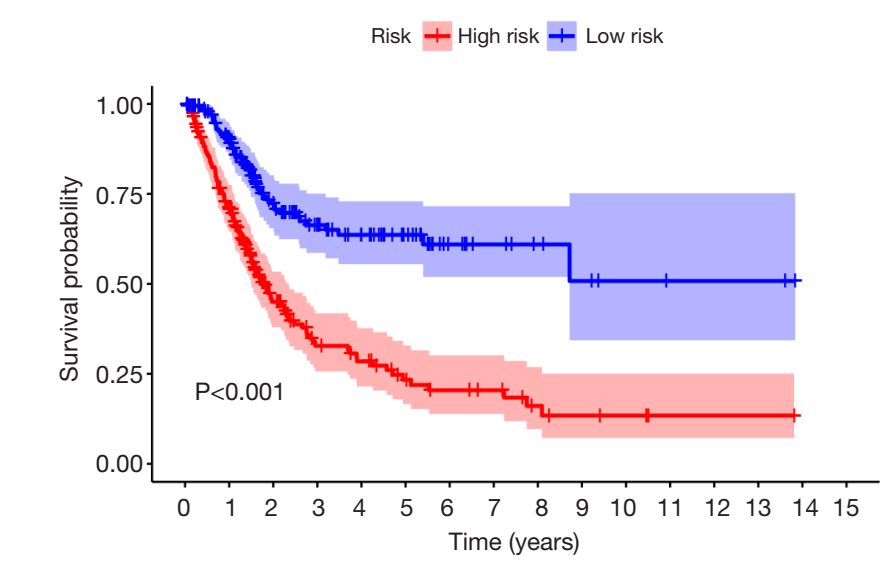

D

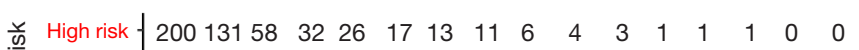

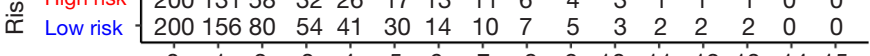
Time (years)

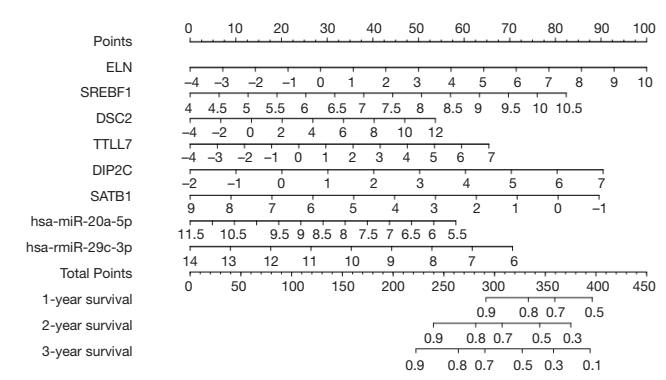

$\mathrm{F}$

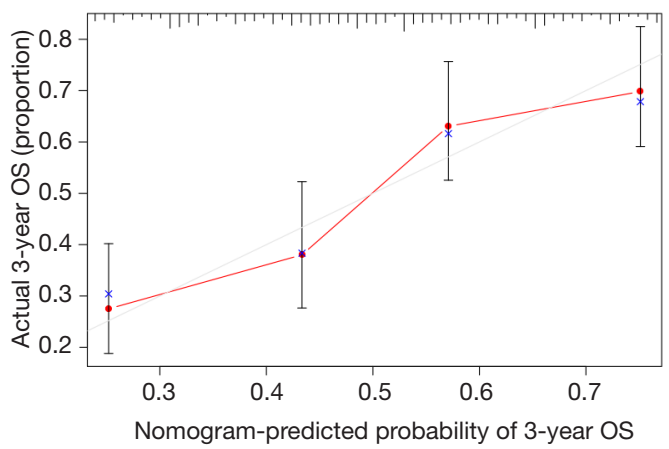

Figure 4 The ceRNA network signature. The ceRNA signature was constructed based on the components of the ceRNA network associated with survival according to LASSO Cox regression analysis (A,B). The results of ROC curve (C), Kaplan-Meier survival curve (D), nomogram (E), and calibration curve (F) analyses are shown. CeRNA, competitive endogenous RNA; LASSO, least absolute shrinkage and selection operator; ROC, receiver operating characteristic.

(Figure $5 A, B)$. The proportions of naive $\mathrm{B}$ cells $(\mathrm{P}=0.001)$, M0 macrophages $(\mathrm{P}<0.001)$, M1 macrophages $(\mathrm{P}<0.001)$, and resting mast cells $(\mathrm{P}=0.012)$ were different in the tumor and normal tissues (Figure 5C). Analysis of correlations between DEG expression and immune cell infiltration in $\mathrm{BC}$ and normal tissues (Figure $5 D$ ) indicated that high levels of CD4 memory-activated T cells $(\mathrm{P}=0.033)$ and CD8 T cells $(\mathrm{P}=0.014)$ were associated with favorable OS, and high levels of memory $\mathrm{B}$ cells were associated with unfavorable survival $(\mathrm{P}=0.002)$ (Figure $5 E, F, G)$. Additionally, we evaluated correlations between clinical and pathological factors and immune cells (Figure 6).

\section{Survival analysis based on immune cells}

All immune cells were incorporated into a univariate Cox regression model to identify immune cells associated with survival $(\mathrm{P}<0.05)($ Table 4). LASSO Cox regression was used 
Table 3 The coefficients of included genes obtained from LASSO Cox regression

\begin{tabular}{lc}
\hline Gene & Coefficient \\
\hline PARVB & 0.0962 \\
RAP1B & 0.0529 \\
PIK3CA & -0.0132 \\
PGF & -0.0604 \\
VEGFA & 0.0294 \\
SDC1 & 0.1073 \\
SPP1 & 0.1006 \\
FLNC & 0.0940 \\
\hline
\end{tabular}

LASSO, least absolute shrinkage and selection operator.

for survival analysis to avoid overfitting (Figure $7 A, B$ ) (Table 5). The median value of the risk score $(0.805)$ calculated by LASSO was used as the cutoff value to divide patients into the high-risk and low-risk groups. ROC curves indicated that OS of BC patients was perfectly predicted by the risk score (AUC for 1 -year survival $=0.704$; AUC for 3 -year survival $=0.666$; and AUC for 5 -year survival $=0.648)($ Figure $7 C)$. The prognosis of $\mathrm{BC}$ patients in the high-risk group was worse than that in the low-risk group $(\mathrm{P}=0.004)$ (Figure $7 D)$. A nomogram predicted the survival probability of 1-, 3-, and 5 -year OS (Figure 7E). The calibration curve confirmed that prediction using the nomogram was accurate (Figure $7 F$ ). Finally, correlations between the components of the ceRNA signature and immune cell signature were analyzed (Figure 8A). Tubulin tyrosine ligase like 7 (TTLL7) and resting mast cells $(\mathrm{R}=0.24, \mathrm{P}<0.001)$, desmocollin 2 (DSC2) and resting mast cells $(\mathrm{R}=-0.23, \mathrm{P}<0.001)$, elastin $(\mathrm{ELN})$ and resting mast cells $(\mathrm{R}=0.44, \mathrm{P}<0.001)$, and hsa-miR-29c-3p and M0 macrophages $(\mathrm{R}=-0.29, \mathrm{P}<0.001)$ had significant correlations (Figure 8B,C,D,E).

\section{Multidimensional validation}

Multidimensional verification included a series of external databases (GEO, GEPIA, HPA, and TargetScan database) to verify the characteristics of significant factors in the coexpression test and survival analysis based on immune cells. Four GEO cohorts were used, including GSE13507, GSE7476, GSE32894, and GSE31684. The results obtained using the TCGA database indicated that the expression of ELN and TTLL7 was higher in normal tissues and the expression of DSC2 was higher in BC tissues. Analysis using the GEPIA database confirmed that both ELN and TTLL7 were expressed at a low level in tumor tissues, and the expression of DSC2 was upregulated. Analysis of the GSE13507 cohort indicated a lack of significant differences in the expression of ELN and DSC2, and the expression of TTLL7 was decreased in BC tissues compared with that in normal tissues. In the GSE7476 cohort, DSC2 was overexpressed and the expression of TTLL7 was decreased in BC tissues; however, the expression of ELN was not significantly different in tumor and normal tissues. The above results were summarized in Table 6. HPA database search results showed that the expression levels of ELN, DSC2, and TTLL7 were significantly increased in BC tissues (Figure 9A,B,C,D,E,F). Analysis of the data of multiple databases (TCGA and GSE31894) indicated that high levels of ELN, DSC2, and TTLL7 were significantly associated with poor prognosis of BC patients (Figure $10 A, B, C, D, E, F)$. Finally, we used GSE31684 to validate the survival analysis based on immune cells. The prognosis of the high-risk group was worse than that of the lowrisk group $(\mathrm{P}<0.001)$ (Figure $10 G)$. A nomogram was able to predict the survival probability of $1-, 3$-, and 5 -year OS (Figure 10H). The calibration curve indicated that prediction using the nomogram was accurate (Figure 10I). Unfortunately, we were unable to verify the survival analysis based on the components of the ceRNA network due to a lack of databases that include mRNA, miRNA, lncRNA, and complete clinical data, which were present only in the TCGA database. Query of the TargetScan database identified binding sites for hsa-miR-29c-3p in ELN and DSC2, and a binding site for hsa-miR-374b-5p was identified in TTLL7 in the ceRNA network (Figure 107).

\section{Discussion}

The number of estimated new BC cases reported in 2019 was 80,470 , and 17,670 BC-related deaths were registered. $\mathrm{BC}$ is characterized by complex biological behavior, a high rate of metastasis, and a high recurrence rate $(40,41)$. After diagnosis, the physical, mental, and social health-related quality of life (HRQoL) of patients with BC significantly declines (42). Increasing evidence indicated that ceRNAs and dysregulation of the immune system are involved in the progression of human tumors (43-48).

Two survival analyses were performed in the present study based on the selected components of a ceRNA network (including ELN, SREBF1, DSC2, TTLL7, DIP2C, SATB1, hsa-miR-20a-5p, and hsa-miR-29c-3p) 


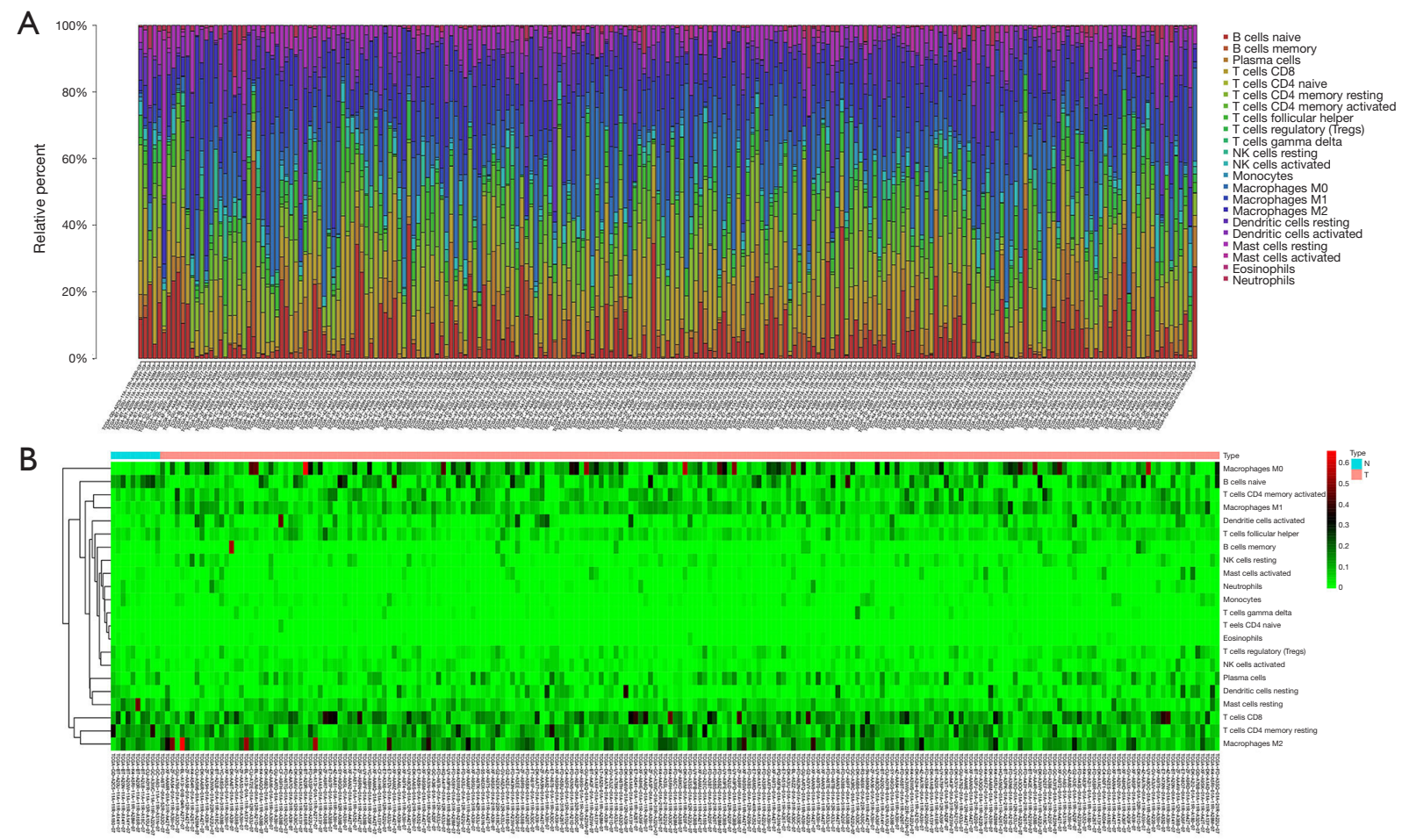

C

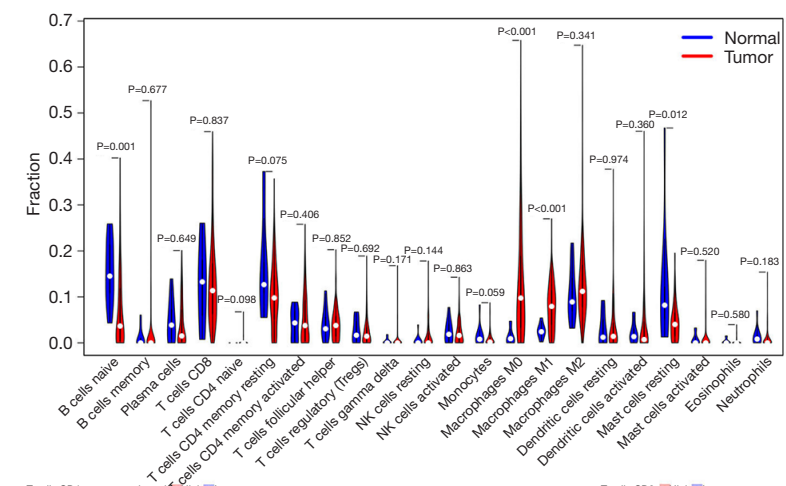

D

$E$

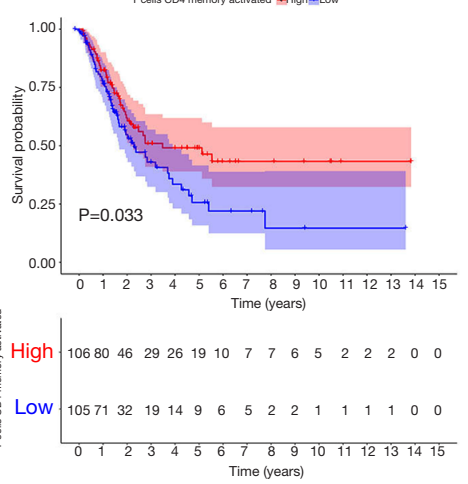

$\mathrm{F}$
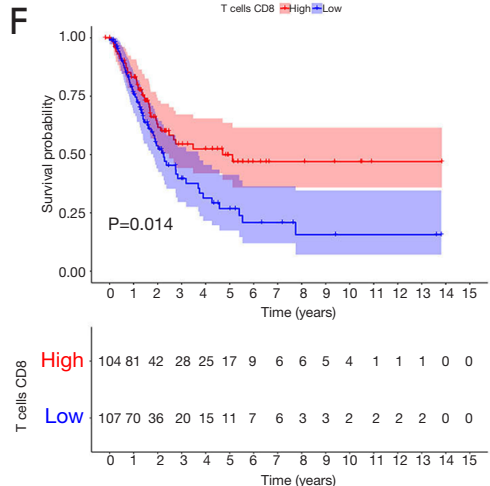

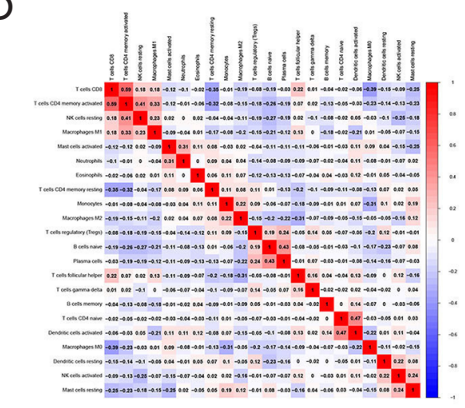

G
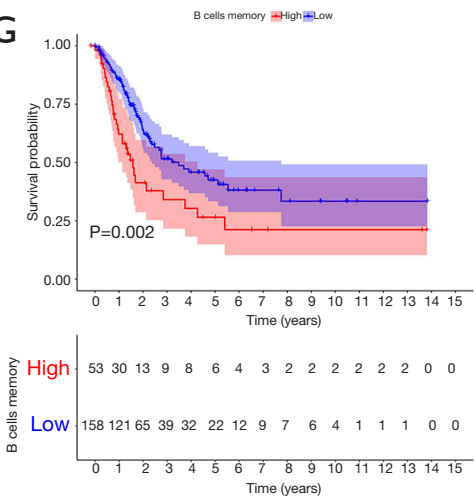

Figure 5 Immune cell infiltration. The composition of immune cells was assessed by the CIBERSORT algorithm in BC (A,B). The proportions of naive B cells, M0 macrophages, M1 macrophages, and resting mast cells were different in BC and normal tissues (C). The results of the correlation analysis between DEG expression and immune cell infiltration are shown as a heatmap (D). High level of activated memory CD4 T cells (E) and CD8 T cells (F) was associated with favorable OS, and a high level of memory B cells was associated with unfavorable OS (G). CIBERSORT, cell-type identification by estimating relative subsets of RNA transcripts; BC, bladder cancer; OS, overall survival. 

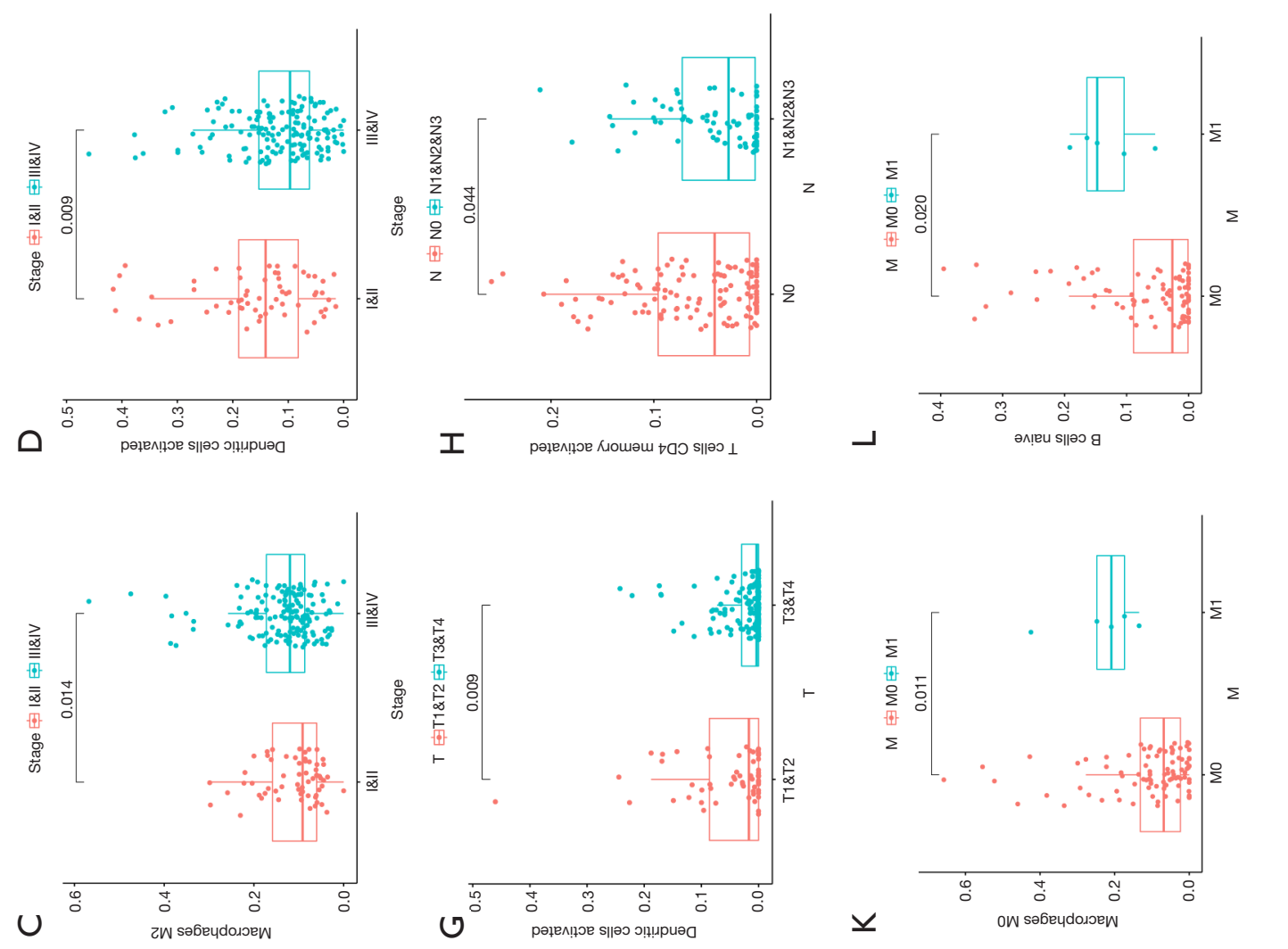

政
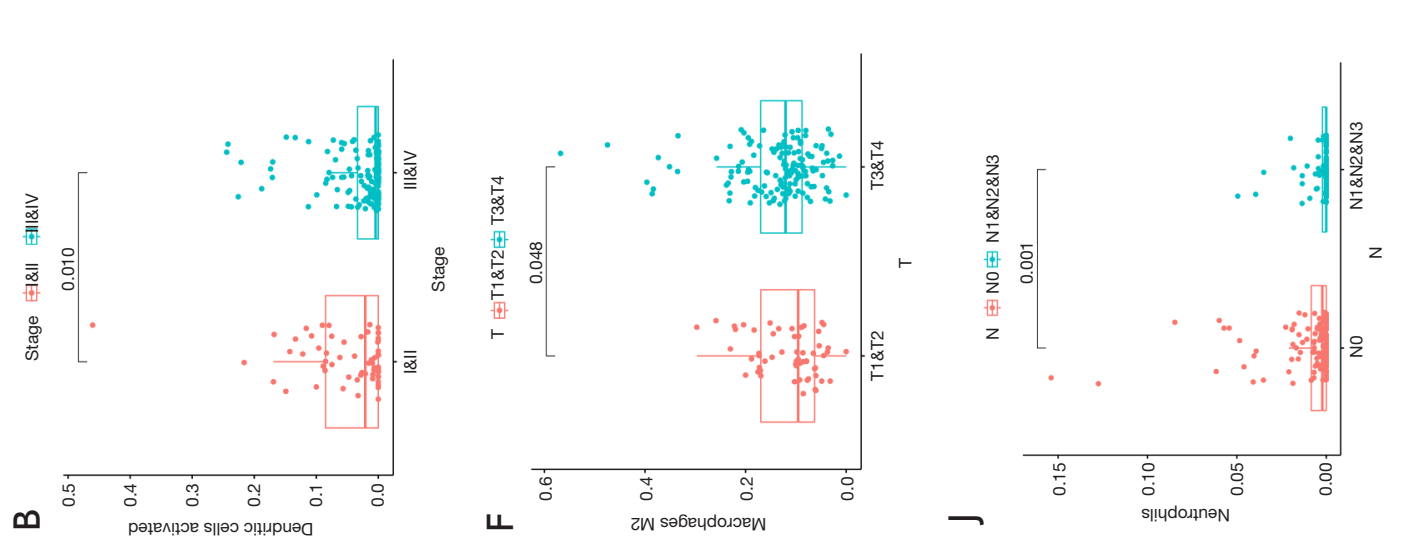

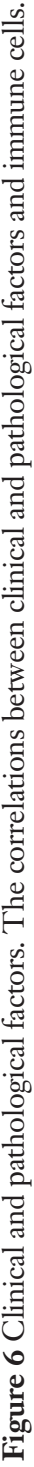


Table 4 The immune cells related to survival identified by univariable Cox regression

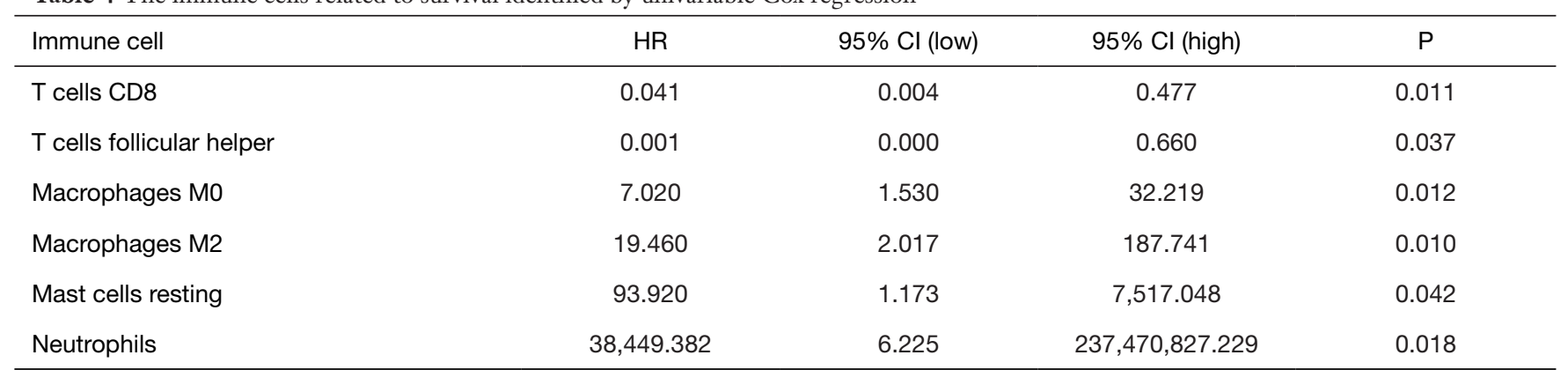

$\mathrm{HR}$, hazard ratio; $\mathrm{Cl}$, confidence interval.

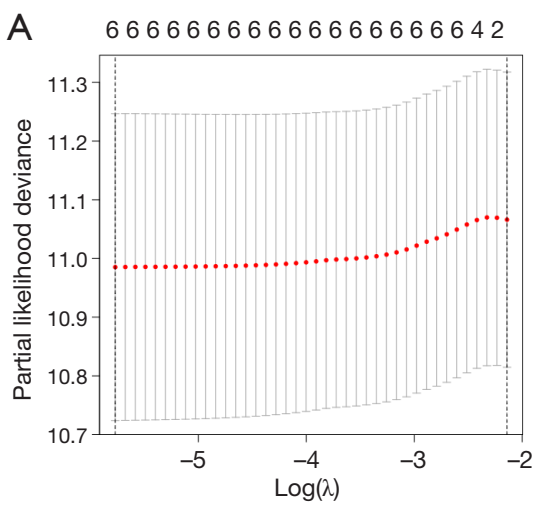

D

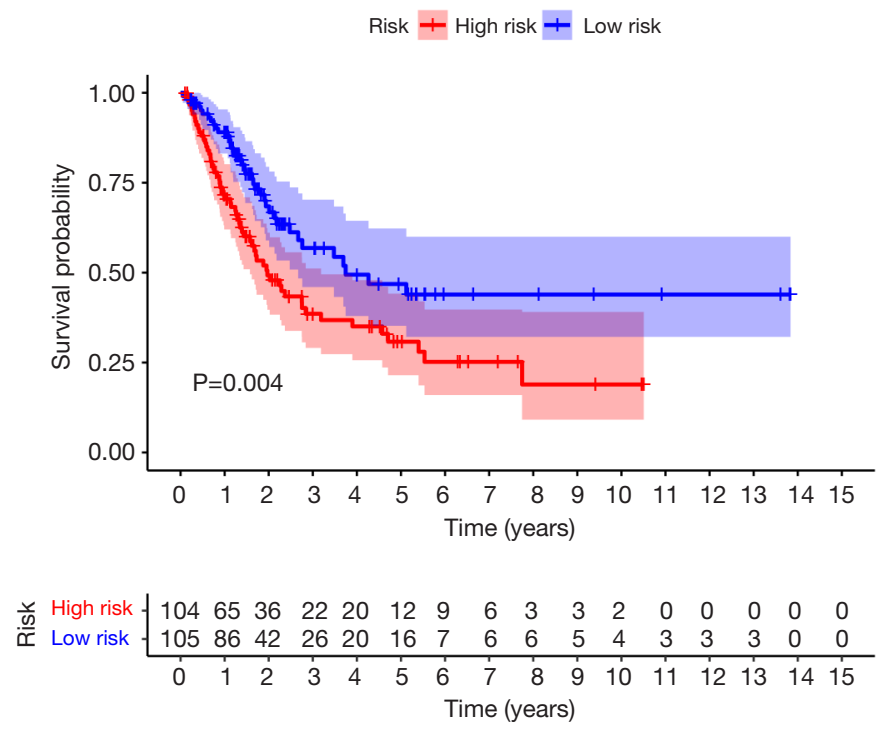

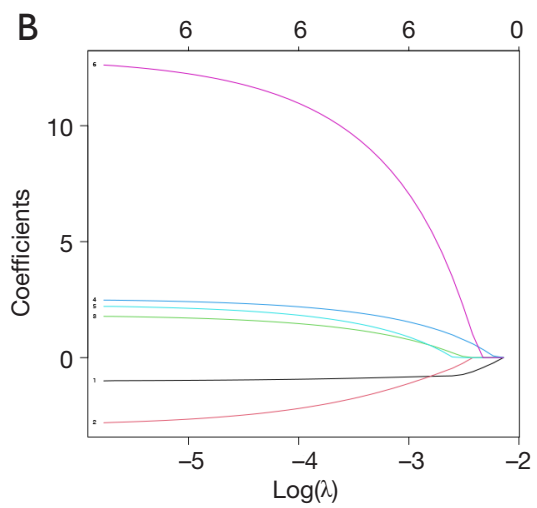

E

Points
C

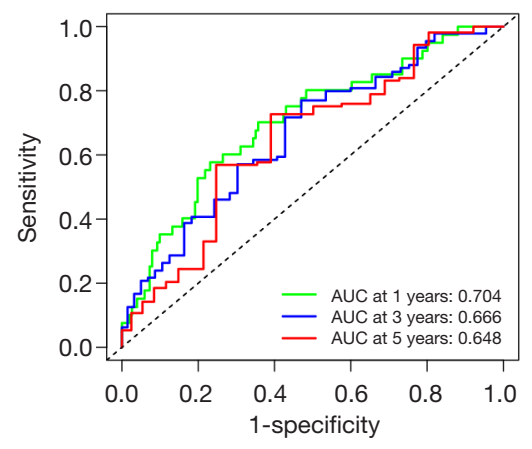

\begin{tabular}{lllllllllll}
0 & 10 & 20 & 30 & 40 & 50 & 60 & 70 & 80 & 90 & 100 \\
\hline
\end{tabular}

\begin{tabular}{lllllllll}
\hline 0.05 & 0.15 & 0.25 & 0.35 & 0.45 & 0.55 & 0.65 \\
\hline
\end{tabular}

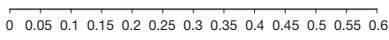
$\begin{array}{llllll}0 & 0.04 & 0.1 & 1 & 0.14 \\ 0 & 0.2 & 0.2\end{array}$

Mast cells resting

Neutrophils

\begin{tabular}{lllllllll}
\hline 0 & 0.02 & 0.04 & 0.06 & 0.08 & 0.1 & 0.12 & 0.14 & 0.16
\end{tabular}

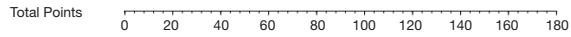

$\begin{array}{lllllll}\text { 1-year survival } & 0.9 & 0.8 & 0.7 & 0.5 & 0.3 & 0.1\end{array}$

3-year survival

5-year survival

$\mathrm{F}$

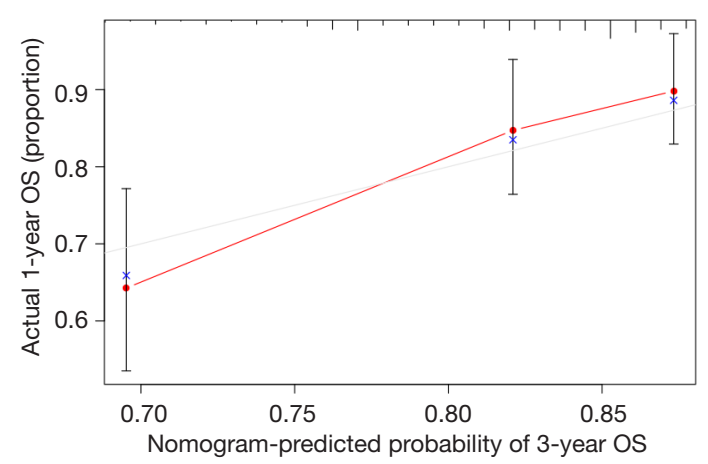

Figure 7 The immune cell signature. The immune cell signature was generated by LASSO Cox regression analysis based on immune cells (A,B). The results of ROC curve (C), Kaplan-Meier survival curve (D), nomogram (E), and calibration curve (F) analyses are shown. LASSO, least absolute shrinkage and selection operator; ROC, receiver operating characteristic. 
and selected immune cells (including M0 macrophages, M2 macrophages, resting mast cells, and neutrophils). Overexpression of SREBF1 was associated with a poor prognosis and promoted metastasis of esophageal carcinoma cells, and inhibition of SREBF1 augmented

Table 5 The coefficients of included immune cells obtained from LASSO Cox regression

\begin{tabular}{lcc}
\hline Immune cell & Coefficient & $\mathrm{P}$ \\
\hline Macrophages M0 & 2.229 & 0.006 \\
Macrophages M2 & 3.022 & 0.014 \\
Mast cells resting & 3.386 & 0.137 \\
Neutrophils & 13.294 & 0.004 \\
\hline
\end{tabular}

LASSO, least absolute shrinkage and selection operator. the efficacy of immune checkpoint blockades $(49,50)$. Downregulated expression of DIP2C was detected in breast cancer, especially in basal-like and HER-2 subtypes (51). Numerous studies confirmed that SATB1 is involved in the occurrence and development of various human tumors (52-55). Hsa-miR-20a-5p inhibits epithelial mesenchymal transition (EMT) and invasion of tumor cells by targeting STAT3 in endometrial cancer (56). The infiltration of M2 macrophages expressing the CD163 antigen decreases the disease-free survival (DFS) rate in high-grade oral tongue squamous cell carcinoma and increases the mortality rate of patients with prostate cancer $(57,58)$. The interaction between neutrophils and cancer is complex. Neutrophil-mediated tumor inflammation can promote the proliferation, invasion, and angiogenesis of tumor cells; however, some studies demonstrated that compounds

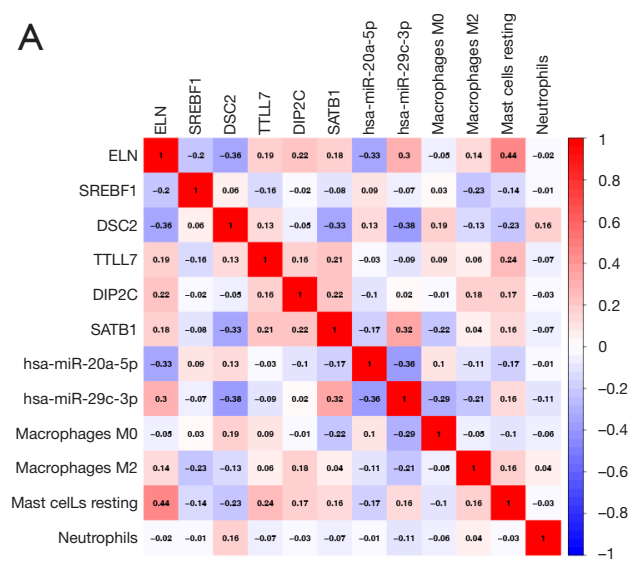

B

C
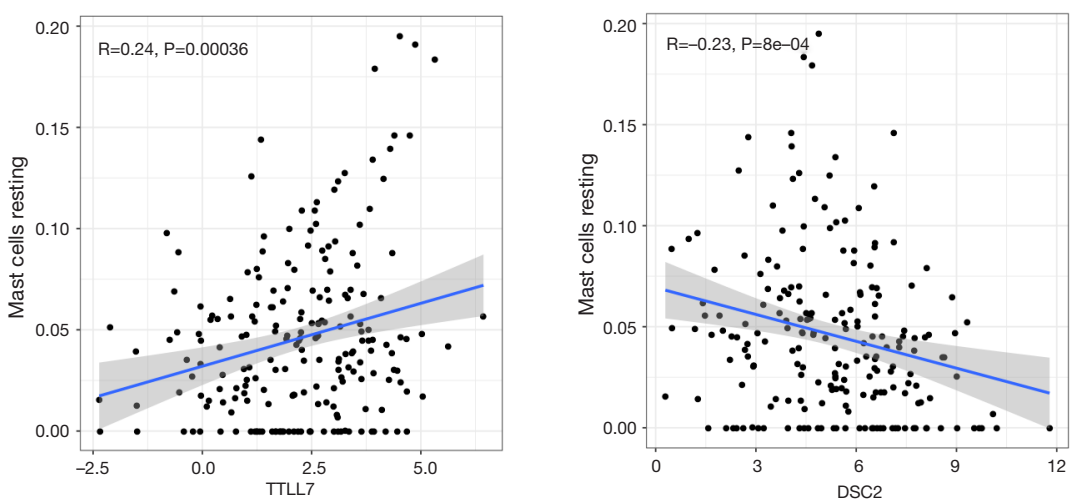

D

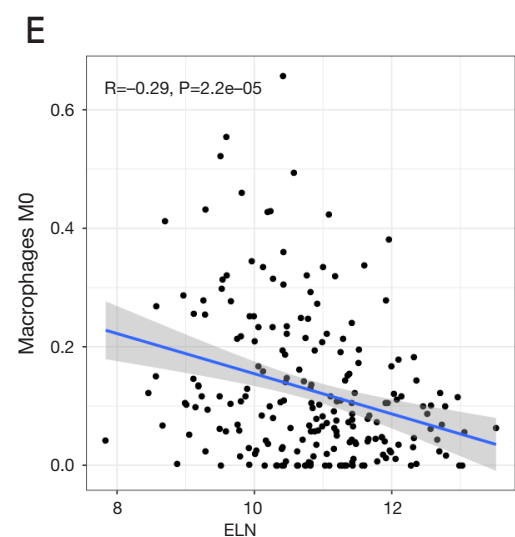

Figure 8 Coexpression analysis. The results of the correlation analysis of DEGs expression and immune cell infiltration are shown as a heatmap (A). The correlations between TTLL7 and resting mast cells (B), DSC2 and resting mast cells (C), ELN and resting mast cells (D), and hsamiR-29c-3p and M0 macrophages (E) were significant. ELN, elastin; DSC2, desmocollin 2; TTLL7, tubulin tyrosine ligase like 7; DEGs, differentially expressed genes. 
Table 6 Multidimensional validation

\begin{tabular}{|c|c|c|c|}
\hline Database & Gene & Tumor vs. normal & $P$ \\
\hline \multirow[t]{3}{*}{ TCGA } & $E L N$ & Down regulated & $<0.001$ \\
\hline & $D S C 2$ & Up regulated & $<0.001$ \\
\hline & $T T L L 7$ & Down regulated & $<0.001$ \\
\hline \multirow[t]{3}{*}{ GEPIA } & $E L N$ & Down regulated & $<0.001$ \\
\hline & $D S C 2$ & Up regulated & $<0.001$ \\
\hline & $T T L L 7$ & Down regulated & $<0.001$ \\
\hline \multirow[t]{3}{*}{ GSE13507 (GEO) } & $E L N$ & NS & 0.184 \\
\hline & $D S C 2$ & NS & 0.687 \\
\hline & $T T L L 7$ & Down regulated & $<0.001$ \\
\hline \multirow[t]{3}{*}{ GSE7476 (GEO) } & $E L N$ & Down regulated & 0.009 \\
\hline & $D S C 2$ & Up regulated & 0.036 \\
\hline & $T T L L 7$ & Down regulated & 0.009 \\
\hline
\end{tabular}

TCGA, the The Cancer Genome Atlas; GEPIA, Gene Expression Profiling Interactive Analysis; GEO, Gene Expression Omnibus; NS, no significance.

produced by neutrophils can suppress tumor development (59-61).

The present study demonstrated significant correlations between TTLL7 and resting mast cells, DSC2 and resting mast cells, ELN and resting mast cells, and hsa-miR-29c$3 p$ and M0 macrophages. Another study suggested that DS2 can be used as a new immunohistochemical biomarker for urothelial carcinoma (UC) with squamous differentiation; this cancer is more advanced and has worse prognosis than those in UC without squamous differentiation (62). In breast cancer, prostate cancer, and lung cancer, increased levels of mast cell infiltration were associated with improved survival rate of patients (63). Decreased expression of hsa-miR-29c-3p was associated with advanced clinical and pathological factors and poor prognosis in laryngeal squamous cell carcinoma, and infiltration of M0 macrophages was associated with poor OS, suggesting a negative correlation between these factors in tumors $(64,65)$. Unfortunately, other components of ceRNA signature were rarely investigated in tumors, and previous studies did not assess possible relationships of these components with immune cells.

The present study has certain limitation. First, our data were obtained from public databases, and clinical and pathological factors included in these databases were
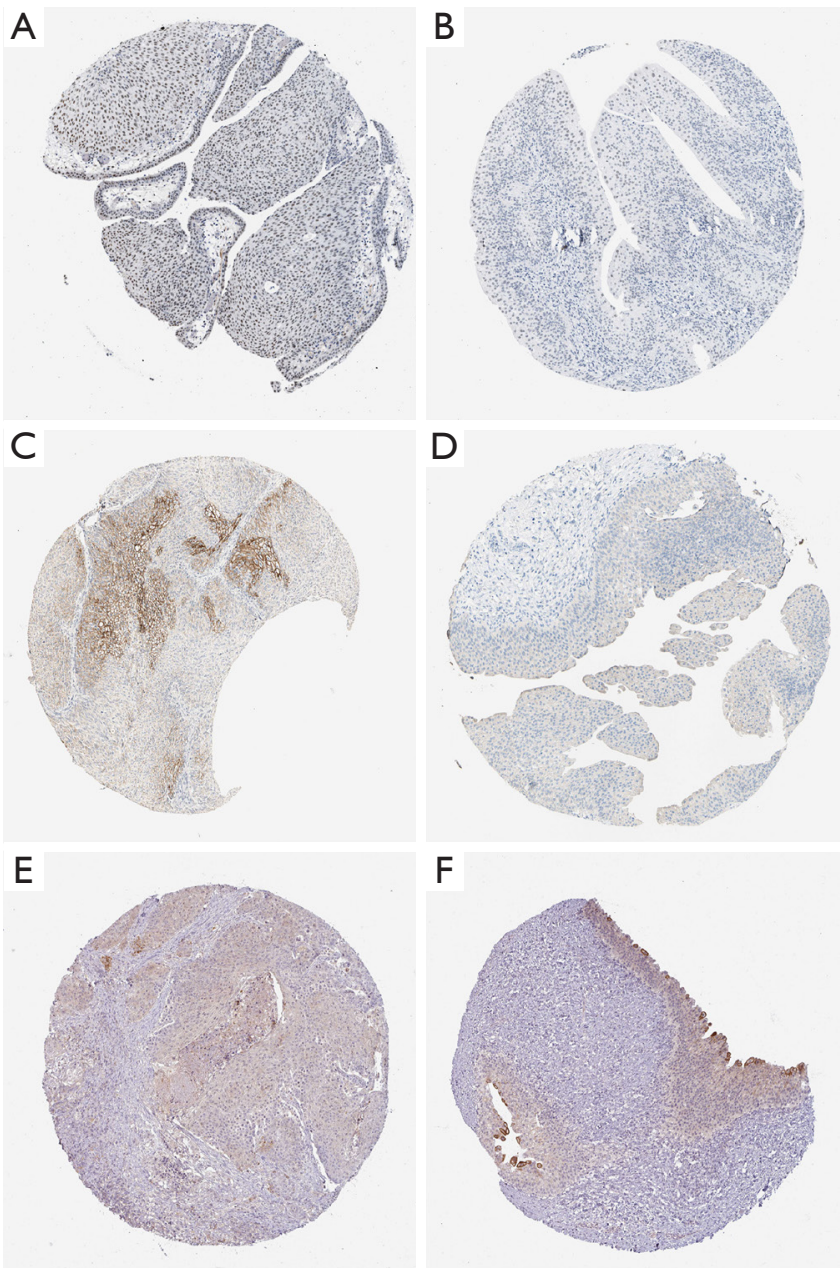

E

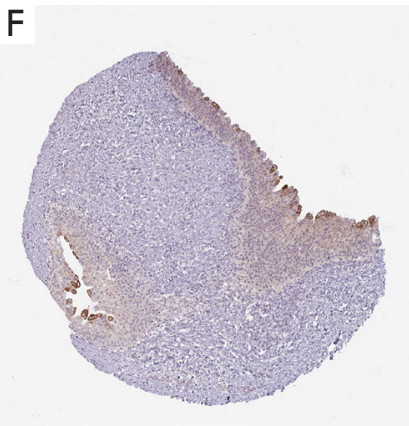

Figure 9 HPA. HPA database search results showed that the expression levels of ELN (A,B), DSC2 (C,D), and TTLL7 (E,F) were significantly increased in BC tissues. HPA, Human Protein Atlas database; ELN, elastin; DSC2, desmocollin 2; TTLL7, tubulin tyrosine ligase like 7; BC, bladder cancer.

not comprehensive; the number of tumor tissue samples was different from the number of normal tissue samples. Second, specific mechanism of infiltration of various immune cell types was not studied in detail. Third, the correlations between the components of the ceRNA network signature and the immune infiltrating cell signature were not confirmed in experiments.

Thus, two survival analyses performed in the present study were based on the selected components of the ceRNA network and selected immune cell types and were used to predict the prognosis of patients with $\mathrm{BC}$; the nomograms containing both of these signatures may provide assistance 
A
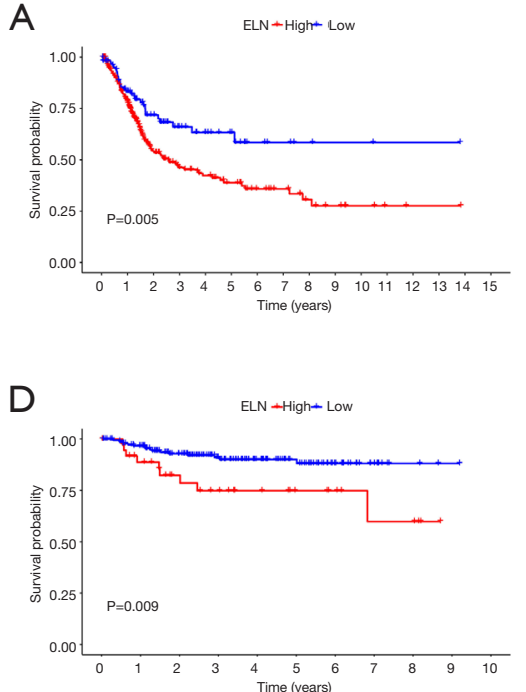

G

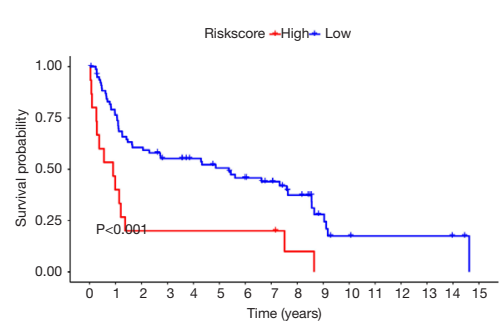

I

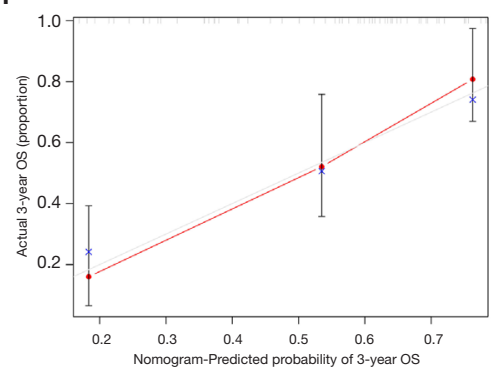

B

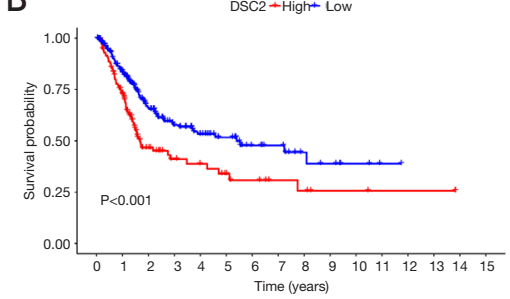

E

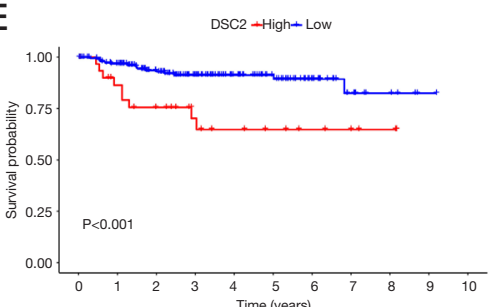

C TLL7+Hight Low

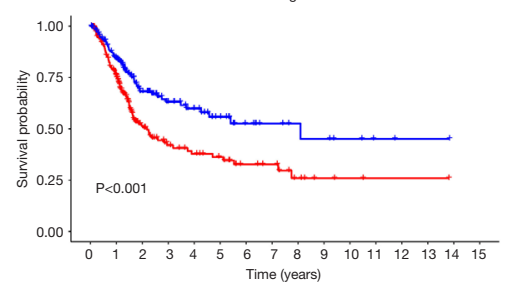

$\mathrm{F}$

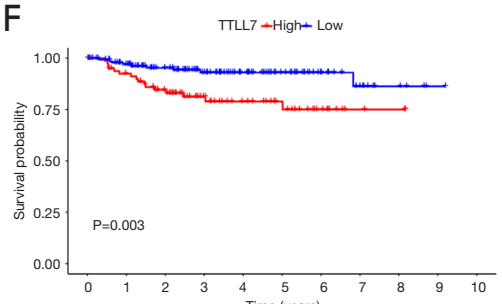

$\mathrm{H}$

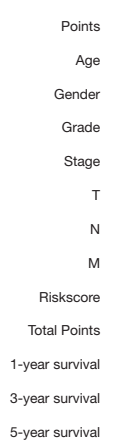

J
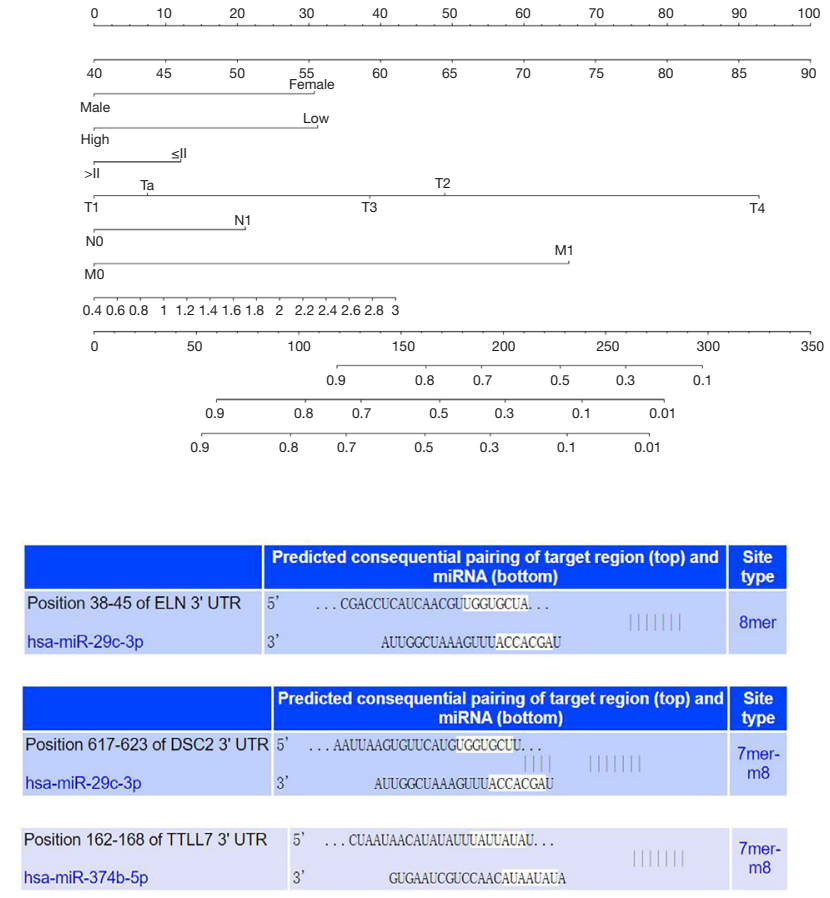

Figure 10 Analysis of the data of multiple databases (TCGA and GSE31894). High levels of ELN, DSC2, and TTLL7 were significantly associated with poor prognosis of BC patients (A-F). Finally, we used GSE31684 to validate the survival analysis based on immune cells. The prognosis of the high-risk group was worse than that of the low-risk group (G). A nomogram was able to predict the survival probability of 1-year, 3-year, and 5-year OS (H). The calibration curve indicated that prediction using the nomogram was accurate (I). Query of the TargetScan database identified binding sites for hsa-miR-29c-3p in ELN and DSC2, and a binding site for hsa-miR-374b-5p was identified in TTLL7 in the ceRNA network (J). ELN, elastin; DSC2, desmocollin 2; TTLL7, tubulin tyrosine ligase like 7; BC, bladder cancer; OS, overall survival. 
to clinicians to improve individual management of $\mathrm{BC}$ patients. Moreover, significant correlations between TTLL7 and resting mast cells, DSC2 and resting mast cells, ELN and resting mast cells, and hsa-miR-29c$3 p$ and M0 macrophages were detected, suggesting that these correlations play significant roles in the prognosis of BC. The present study provides some useful information for prediction of the prognosis of $\mathrm{BC}$ patients; however, further studies are needed to clarify the relationships between ceRNAs and immune infiltrating cells and relevant molecular mechanisms.

\section{Acknowledgments}

Funding: This work was supported by the Project of Liaoning Distinguished Professor [grant no. [2012]145] for KCZ, the Shenyang Plan Project of Science and Technology [grant no. F17-230-9-08] for KCZ, China Medical University 2017 Discipline Promotion Program [grant no. 3110117040] for KCZ, China Medical University 2018 Discipline Promotion Program for KCZ, and the 2017 National Key R\&D Program Key Projects of Precision Medical Research [no. 2017YFC0908000] for KCZ.

\section{Footnote}

Reporting Checklist: The authors have completed the STROBE reporting checklist. Available at http://dx.doi. org/10.21037/tau-20-1250

Conflicts of Interest: The authors have completed the ICMJE uniform disclosure form (available at http://dx.doi. org/10.21037/tau-20-1250). The authors have no conflicts of interest to declare.

Ethical Statement: The authors are accountable for all aspects of the work in ensuring that questions related to the accuracy or integrity of any part of the work are appropriately investigated and resolved. The study was conducted in accordance with the Declaration of Helsinki (as revised in 2013).

Open Access Statement: This is an Open Access article distributed in accordance with the Creative Commons Attribution-NonCommercial-NoDerivs 4.0 International License (CC BY-NC-ND 4.0), which permits the noncommercial replication and distribution of the article with the strict proviso that no changes or edits are made and the original work is properly cited (including links to both the formal publication through the relevant DOI and the license). See: https://creativecommons.org/licenses/by-nc-nd/4.0/.

\section{References}

1. Siegel R, Naishadham D, Jemal A. Cancer statistics, 2013. CA Cancer J Clin 2013;63:11-30.

2. Bray F, Ferlay J, Soerjomataram I, et al. Global cancer statistics 2018: GLOBOCAN estimates of incidence and mortality worldwide for 36 cancers in 185 countries. CA Cancer J Clin 2018;68:394-424.

3. Cheng $\mathrm{S}$, Jiang $Z$, Xiao J, et al. The prognostic value of six survival-related genes in bladder cancer. Cell Death Discov 2020;6:58.

4. Wang Y, Chen L, Yu M, et al. Immune-related signature predicts the prognosis and immunotherapy benefit in bladder cancer. Cancer Med 2020;9:7729-41.

5. Nabavizadeh R, Bobrek K, Master VA. Risk stratification for bladder cancer: Biomarkers of inflammation and immune activation. Urol Oncol 2020;38:706-12.

6. Li F, Guo H, Wang Y, et al. Profiles of tumorinfiltrating immune cells and prognostic genes associated with the microenvironment of bladder cancer. Int Immunopharmacol 2020;85:106641.

7. Chen X, Jin Y, Gong L, et al. Bioinformatics Analysis Finds Immune Gene Markers Related to the Prognosis of Bladder Cancer. Front Genet 2020;11:607.

8. Lucca I, de Martino M, Klatte T, et al. Novel biomarkers to predict response and prognosis in localized bladder cancer. Urol Clin North Am 2015;42:225-33, ix.

9. Zhang XQ, Sun S, Lam KF, et al. A long non-coding RNA signature in glioblastoma multiforme predicts survival. Neurobiol Dis 2013;58:123-31.

10. Wang H, Niu X, Jiang H, et al. Long non-coding RNA DLX6-AS1 facilitates bladder cancer progression through modulating miR-195-5p/VEGFA signaling pathway. Aging (Albany NY) 2020;12:16021-34.

11. Li C, Cao Y, Zhang L, et al. LncRNA IGFBP4-1 promotes tumor development by activating Janus kinase-signal transducer and activator of transcription pathway in bladder urothelial carcinoma. Int J Biol Sci 2020;16:2271-82.

12. Li Q, Huyan T, Cai S, et al. The role of exosomal miR375-3p: A potential suppressor in bladder cancer via the Wnt//-catenin pathway. FASEB J 2020;34:12177-96.

13. Cai X, Qu L, Yang J, et al. Exosome-transmitted microRNA-133b inhibited bladder cancer proliferation 
by upregulating dual-specificity protein phosphatase 1 . Cancer Med 2020;9:6009-19.

14. Zhu X, Jiang L, Yang H, et al. Analyzing the lncRNA, miRNA, and mRNA-associated ceRNA networks to reveal potential prognostic biomarkers for glioblastoma multiforme. Cancer Cell Int 2020;20:393.

15. Salmena L, Poliseno L, Tay Y, et al. A ceRNA hypothesis: the Rosetta Stone of a hidden RNA language? Cell 2011;146:353-8.

16. Long J, Bai Y, Yang X, et al. Construction and comprehensive analysis of a ceRNA network to reveal potential prognostic biomarkers for hepatocellular carcinoma. Cancer Cell Int 2019;19:90.

17. Jiang Y, Wang J, Chen J, et al. Construction and analysis of an aberrant IncRNA-miRNA-mRNA network associated with papillary thyroid cancer. Medicine (Baltimore) 2020;99:e22705.

18. Wang Z, Li Y, Zhong Y, et al. Comprehensive Analysis of Aberrantly Expressed Competitive Endogenous RNA Network and Identification of Prognostic Biomarkers in Pheochromocytoma and Paraganglioma. Onco Targets Ther 2020;13:11377-95.

19. Carter BW, Halpenny DF, Ginsberg MS, et al. Immunotherapy in Non-Small Cell Lung Cancer Treatment: Current Status and the Role of Imaging. J Thorac Imaging 2017;32:300-12.

20. Lin P, Guo YN, Shi L, et al. Development of a prognostic index based on an immunogenomic landscape analysis of papillary thyroid cancer. Aging (Albany NY) 2019;11:480-500.

21. Kresowik TP, Griffith TS. Bacillus Calmette-Guerin immunotherapy for urothelial carcinoma of the bladder. Immunotherapy 2009;1:281-8.

22. Pettenati C, Ingersoll MA. Mechanisms of BCG immunotherapy and its outlook for bladder cancer. Nat Rev Urol 2018;15:615-25.

23. Herr HW, Morales A. History of bacillus CalmetteGuerin and bladder cancer: an immunotherapy success story. J Urol 2008;179:53-6.

24. Seidl C. Targets for Therapy of Bladder Cancer. Semin Nucl Med 2020;50:162-70.

25. Laurberg JR, Jensen JB, Schepeler T, et al. High expression of GEM and EDNRA is associated with metastasis and poor outcome in patients with advanced bladder cancer. BMC Cancer 2014;14:638.

26. Walker MP, Zhang M, Le TP, et al. RAC3 is a promigratory co-activator of ER $\alpha$. Oncogene 2011;30:1984-94.

27. Gust KM, Rebhan K, Resch I, et al. Immune checkpoint inhibition in muscle-invasive and locally advanced bladder cancer. Curr Opin Urol 2020;30:547-56.

28. Zhu N, Hou J, Wu Y, et al. Integrated analysis of a competing endogenous RNA network reveals key lncRNAs as potential prognostic biomarkers for human bladder cancer. Medicine (Baltimore) 2018;97:e11887.

29. Galon J, Costes A, Sanchez-Cabo F, et al. Type, density, and location of immune cells within human colorectal tumors predict clinical outcome. Science 2006;313:1960-4.

30. Huang R, Wu J, Zheng Z, et al. The Construction and Analysis of ceRNA Network and Patterns of Immune Infiltration in Mesothelioma With Bone Metastasis. Front Bioeng Biotechnol 2019;7:257.

31. Huang R, Meng T, Chen R, et al. The construction and analysis of tumor-infiltrating immune cell and ceRNA networks in recurrent soft tissue sarcoma. Aging (Albany NY) 2019;11:10116-43.

32. Love MI, Huber W, Anders S. Moderated estimation of fold change and dispersion for RNA-seq data with DESeq2. Genome Biol 2014;15:550.

33. Kang K, Xie F, Mao J, et al. Significance of Tumor Mutation Burden in Immune Infiltration and Prognosis in Cutaneous Melanoma. Front Oncol 2020;10:573141.

34. Zhang J, Lou W. A Key mRNA-miRNA-lncRNA Competing Endogenous RNA Triple Sub-network Linked to Diagnosis and Prognosis of Hepatocellular Carcinoma. Front Oncol 2020;10:340.

35. Li JH, Liu S, Zhou H, et al. starBase v2.0: decoding miRNA-ceRNA, miRNA-ncRNA and protein-RNA interaction networks from large-scale CLIP-Seq data. Nucleic Acids Res 2014;42:D92-7.

36. Chang Z, Huang R, Fu W, et al. The Construction and Analysis of ceRNA Network and Patterns of Immune Infiltration in Colon Adenocarcinoma Metastasis. Front Cell Dev Biol 2020;8:688.

37. Newman AM, Liu CL, Green MR, et al. Robust enumeration of cell subsets from tissue expression profiles. Nat Methods 2015;12:453-7.

38. Sun XY, Yu SZ, Zhang HP, et al. A signature of 33 immune-related gene pairs predicts clinical outcome in hepatocellular carcinoma. Cancer Med 2020;9:2868-78.

39. Li R, Qu H, Wang S, et al. GDCRNATools: an R/ Bioconductor package for integrative analysis of lncRNA, miRNA and mRNA data in GDC. Bioinformatics 2018;34:2515-7.

40. Howlader N, Noone AM, Krapcho M, et al. SEER cancer statistics review, 1975-2016, National Cancer Institute. Bethesda, MD. 2019. https://seer.cancer.gov/csr/based 
on November 2018 SEER data submission, posted to the SEER web site.

41. Alfred Witjes J, Lebret T, Compérat EM, et al. Updated 2016 EAU Guidelines on Muscle-invasive and Metastatic Bladder Cancer. Eur Urol 2017;71:462-75.

42. Smith AB, Jaeger B, Pinheiro LC, et al. Impact of bladder cancer on health-related quality of life. BJU Int 2018;121:549-57.

43. Zheng X, Wang X, Zheng L, et al. Construction and Analysis of the Tumor-Specific mRNA-miRNA-lncRNA Network in Gastric Cancer. Front Pharmacol 2020;11:1112.

44. Ma L, Song G, Li M, et al. Construction and Comprehensive Analysis of a ceRNA Network to Reveal Potential Novel Biomarkers for Triple-Negative Breast Cancer. Cancer Manag Res 2020;12:7061-75.

45. Liu S, Song A, Zhou X, et al. ceRNA network development and tumour-infiltrating immune cell analysis of metastatic breast cancer to bone. J Bone Oncol 2020;24:100304.

46. Duan S, Wang P, Liu F, et al. Novel immune-risk score of gastric cancer: A molecular prediction model combining the value of immune-risk status and chemosensitivity. Cancer Med 2019;8:2675-85.

47. Angell H, Galon J. From the immune contexture to the Immunoscore: the role of prognostic and predictive immune markers in cancer. Curr Opin Immunol 2013;25:261-7.

48. Gentles AJ, Newman AM, Liu CL, et al. The prognostic landscape of genes and infiltrating immune cells across human cancers. Nat Med 2015;21:938-45.

49. Huang CM, Huang CS, Hsu TN, et al. Disruption of Cancer Metabolic SREBP1/miR-142-5p Suppresses Epithelial-Mesenchymal Transition and Stemness in Esophageal Carcinoma. Cells 2019;9:7.

50. Liu C, Chikina M, Deshpande R, et al. Treg Cells Promote the SREBP1-Dependent Metabolic Fitness of TumorPromoting Macrophages via Repression of CD8(+) T CellDerived Interferon- $\gamma$. Immunity 2019;51:381-97.e6.

51. Li J, Ping JL, Ma B, et al. DIP2C expression in breast cancer and its clinical significance. Pathol Res Pract 2017;213:1394-9.

52. Mir R, Pradhan SJ, Patil P, et al. Wnt/ $\beta$-catenin signaling regulated SATB1 promotes colorectal cancer tumorigenesis and progression. Oncogene 2016;35:1679-91.

53. Mao LJ, Yang CH, Fan L, et al. SATB1 promotes prostate cancer metastasis by the regulation of epithelialmesenchymal transition. Biomed Pharmacother 2016;79:1-8.
54. Zhai S, Xue J, Wang Z, et al. High expression of special AT-rich sequence binding protein-1 predicts esophageal squamous cell carcinoma relapse and poor prognosis. Oncol Lett 2017;14:7455-60.

55. Chen Z, Li Z, Li W, et al. SATB1 Promotes Pancreatic Cancer Growth and Invasion Depending on MYC Activation. Dig Dis Sci 2015;60:3304-17.

56. Huang Y, Yang N. MicroRNA-20a-5p inhibits epithelial to mesenchymal transition and invasion of endometrial cancer cells by targeting STAT3. Int J Clin Exp Pathol 2018;11:5715-24.

57. Genin M, Clement F, Fattaccioli A, et al. M1 and M2 macrophages derived from THP-1 cells differentially modulate the response of cancer cells to etoposide. BMC Cancer 2015;15:577.

58. Agarbati S, Mascitti M, Paolucci E, et al. Prognostic Relevance of Macrophage Phenotypes in Highgrade Oral Tongue Squamous Cell Carcinomas. Appl Immunohistochem Mol Morphol 2021;29:359-65.

59. Jamieson T, Clarke M, Steele CW, et al. Inhibition of CXCR2 profoundly suppresses inflammation-driven and spontaneous tumorigenesis. J Clin Invest 2012;122:3127-44.

60. Hanahan D, Weinberg RA. Hallmarks of cancer: the next generation. Cell 2011;144:646-74.

61. Fridlender ZG, Sun J, Kim S, et al. Polarization of tumorassociated neutrophil phenotype by TGF-beta: "N1" versus "N2" TAN. Cancer Cell 2009;16:183-94.

62. Hayashi T, Sentani K, Oue N, et al. Desmocollin 2 is a new immunohistochemical marker indicative of squamous differentiation in urothelial carcinoma. Histopathology 2011;59:710-21.

63. Oldford SA, Marshall JS. Mast cells as targets for immunotherapy of solid tumors. Mol Immunol 2015;63:113-24.

64. Fang R, Huang Y, Xie J, et al. Downregulation of miR$29 \mathrm{c}-3 \mathrm{p}$ is associated with a poor prognosis in patients with laryngeal squamous cell carcinoma. Diagn Pathol 2019;14:109.

65. Wang Y, Ba HJ, Liu ZC, et al. Prognostic value of immune cell infiltration in bladder cancer: A gene expression-based study. Oncol Lett 2020;20:1677-84.

Cite this article as: $\mathrm{Fu} \mathrm{Y,} \mathrm{Sun} \mathrm{S,} \mathrm{Bi} \mathrm{J,} \mathrm{Kong} \mathrm{C,} \mathrm{Yin} \mathrm{L.}$ Construction and analysis of a ceRNA network and patterns of immune infiltration in bladder cancer. Transl Androl Urol 2021;10(5):1939-1955. doi: 10.21037/tau-20-1250 